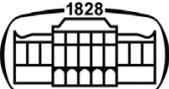

AKADÉMIAI KIADÓ

International Review of

Applied Sciences and Engineering

$11(2020) 1,15-21$

DOI:

$10.1556 / 1848.2020 .00003$

(c) 2020 The Author

\section{ORIGINAL RESEARCH} PAPER

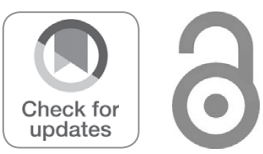

\title{
Analysis of inelastic buckling of rectangular plates with a free edge using polynomial deflection functions
}

\author{
UCHECHI G. EZIEFULA ๑
}

School of Engineering Technology, Imo State Polytechnic Umuagwo, P.M.B. 1472, Owerri, Imo, Nigeria

Received: January 31, 2019 • Accepted: March 20, 2019

Published online: April 24, 2020

\begin{abstract}
The inelastic buckling behaviour of different rectangular thin isotropic plates having a free edge is studied. Various combinations of boundary conditions are subject to in-plane uniaxial compression and each rectangular plate is bounded by an unloaded free edge. The characteristic deflection function of each plate is formulated using a polynomial function in form of Taylor-Maclaurin series. A deformation plasticity approach is adopted and the buckling load equation is modified using a work principle technique. Buckling coefficients of the plates are calculated for various aspect ratios and moduli ratios. Findings obtained from the investigation are found to reasonably agree with data published in the literature.
\end{abstract}

KEYWORDS

deflection function, free edge, plastic buckling, rectangular plate, Taylor's series

\section{INTRODUCTION}

Rectangular plates are used as thin-walled structural elements in engineering structures, such as ships, aircrafts, bridges, and offshore structures. Analysis of inelastic buckling of thin rectangular plates is important, especially if the plate material, such as aluminium alloys and stainless steel, has a low proportional limit in comparison with the nominal yield stress [1]. Inelastic and elasto-plastic buckling of rectangular plates subjected to in-plane compression have been analysed using different methods and plasticity theories. Recent investigations on elasto-plastic and inelastic buckling of plates have been conducted [2-11]. Analytical solutions to elasto-plastic and inelastic buckling problems of rectangular plates are available for simply-supported plates [12]. Closed-form solutions for most boundary conditions are difficult to obtain owing to the mathematical complexities involved. Various energy and numerical approaches such as the Rayleigh-Ritz, Galerkin, finite difference, finite element and differential quadrature methods are, therefore, employed to solve more complex problems. A work principle technique [13] is an approximate method, which uses total work error functional and requires minimisation. The technique is applicable to deflection functions with multiple degrees of freedom and seems to have more effective convergence than the Raleigh-Ritz technique [14]. The work principle is combined with Taylor's series to form the deflection function of rectangular plates. The Taylor's series is more useful than the trigonometric series in formulating deflection functions of various boundary condition arrangements whose solutions are difficult to solve [14].

The inelastic and elasto-plastic buckling of rectangular plates subjected to free edges have not received much attention although some researchers [15-20] analysed plate elastic buckling with free edges. Maarefdoust and Kadkhodayan [7] studied the elasto-plastic buckling of rectangular plates using deformation plasticity theory and generalised differential 


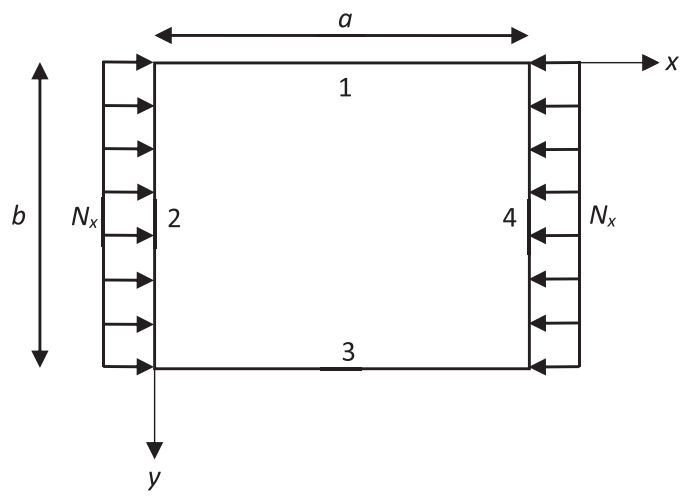

Figure 1. Rectangular plate showing the loading condition and the edge numbers

quadrature method, with each plate having one or two loaded free edges. The inelastic buckling of different isotropic thin rectangular plates with an unloaded free edge is analysed in this article using polynomial deflection functions in the energy approach. Total work error function, deformation theory of plasticity and Taylor-Maclaurin series are applied in deriving the critical buckling loads. Different boundary condition combinations under uniaxial loading are studied.

\section{BOUNDARY CONDITIONS AND POLYNOMIAL DEFLECTION FUNCTIONS}

An isotropic thin rectangular flat plate under in-plane uniaxial compressive loads is considered (Fig. 1). The compressive loads act parallel with the longitudinal direction ( $x$-axis), and the edge numbering is identified as seen in Fig. 1. Six boundary condition combinations are analysed in the present study. The boundary conditions are considered such that each plate has an unloaded free edge. The other three edges are simply supported, clamped, or a blend of both simply-supported and clamped. The combinations of the boundary conditions are SSFS, CCFC, SCFS, SCFC, CSFS and CSFC (Fig. 2). The letters are denoted such that the first letter represents the support condition at edge 1, the second letter is for edge 2, the third letter is for edge 3 and the fourth letter is for edge 4. For example, for the SCFC plate, edge 1 is simply supported, edges 2 and 4 are clamped, whereas edge 3 is free.

A non-dimensional system is obtained from the Cartesian axes to make it easier to solve the problem:

$$
x=a X ; y=b Y
$$

where $a$ and $b$ are the sides of the plate which represent the length and width, respectively while $X$ and $Y$ are nondimensional axes in terms of $x$-axis and $y$-axis, respectively. The boundary conditions for the simply-supported edges at $X=0$ and $X=1$ are as follows:

$$
w=0 ; w_{X X}=0
$$

and are, at $Y=0$ and $Y=1$ :

$$
w=0 ; \quad w_{Y Y}=0
$$

where $w$ is the deflection function, and $w_{X X}$ and $w_{Y Y}$ are the second derivatives of the deflection with respect to the $X$ and $Y$-axes, respectively. Similarly, the boundary conditions for the clamped edges at $X=0$ and $X=1$ are as follows:

$$
w=0 ; w_{X}=0
$$

and, at $Y=0$ and $Y=1$, are:

$$
w=0 ; w_{Y}=0
$$

where $w_{X}$ and $w_{Y}$ are the first derivatives of the deflection with respect to the $X$ - and $Y$-axes, respectively. Repeating the same procedure for free edges gives the boundary conditions at $X=0$ and $X=1$ as follows:

$$
w_{X X}=0 ; w_{X X X}=0
$$

and the boundary conditions at $Y=0$ and $Y=1$ are as follows:
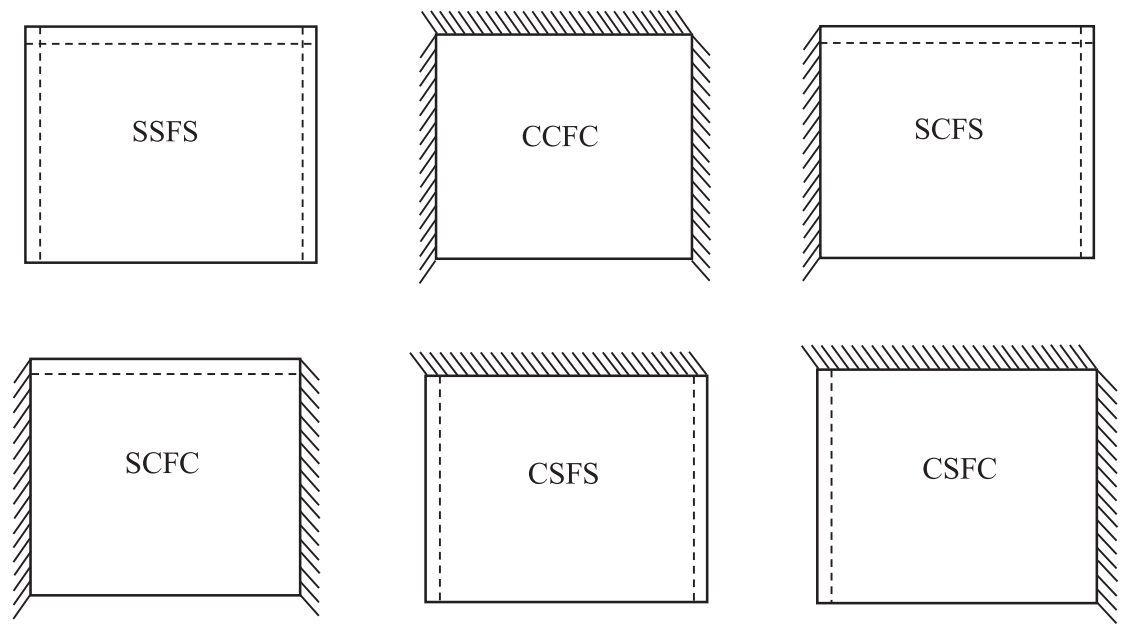

Figure 2. Boundary conditions of rectangular plates) 


$$
w_{Y Y}=0 ; w_{Y Y Y}=0
$$

where $w_{X X X}$ and $w_{Y Y Y}$ are the third derivatives of the deflection with respect to the $X$ - and $Y$-axes, respectively. In the problem definition shown in Fig. 2, the free edge boundary condition is only considered along the edge, $Y=$ 1. Thus, the boundary conditions for the free edge for all the plates being considered are as follows:

$$
w_{Y Y}(Y=1) ; w_{Y Y Y}(Y=1)=0
$$

The Taylor's series refers to any representation of a function, $f_{x}$, as an infinite sum of terms calculated from the values of the derivatives of the function at a single point, $x_{0}$ [21]. Glyn [21] expressed Taylor's series as follows:

$$
f(x)=\sum_{n=0}^{\infty} \frac{\left(x-x_{0}\right)^{n}}{n !} f^{(n)}\left(x_{0}\right)
$$

The Maclaurin series expansion is a special type of Taylor's series when $x_{0}$ is equal to zero. Taking $f_{n}\left(x_{0}\right) / \mathrm{n}$ ! as $\alpha_{n}$ and making $x_{0}$ equal to zero, the Taylor-Maclaurin series may be written as follows:

$f(x)=\alpha_{0}+\alpha_{1} x+\alpha_{2} x^{2}+\alpha_{3} x^{3}+\alpha_{4} x^{4}+\ldots+\alpha_{n} x^{n}$

A polynomial function written in form of Eq. (10) can be used to express the deflection function of a rectangular plate [22]. Using this approach involves applying the nondimensional coordinate system given in Eq. (1) and truncating the polynomial series at the fifth term. The deflection equation, therefore, is expressed as follows:

$$
\begin{aligned}
w= & A\left[( \alpha _ { 0 } + \alpha _ { 1 } X + \alpha _ { 2 } X ^ { 2 } + \alpha _ { 3 } X ^ { 3 } + \alpha _ { 4 } X ^ { 4 } ) \left(\beta_{0}+\beta_{1} Y\right.\right. \\
& \left.\left.+\beta_{2} Y^{2}+\beta_{3} Y^{3}+\beta_{4} Y^{4}\right)\right]
\end{aligned}
$$

where $A$ is the amplitude, $\alpha_{1}, \alpha_{2}, \alpha_{3}$ and $\alpha_{4}$ are unknown constants of the polynomial series with respect to the $X$-direction, and $\beta_{1}, \beta_{2}, \beta_{3}$ and $\beta_{4}$ are unknown constants of the polynomial series with respect to the $Y$-direction. The deflection function (11) can be expressed in terms of the buckling curve expression, $H$, as follows:

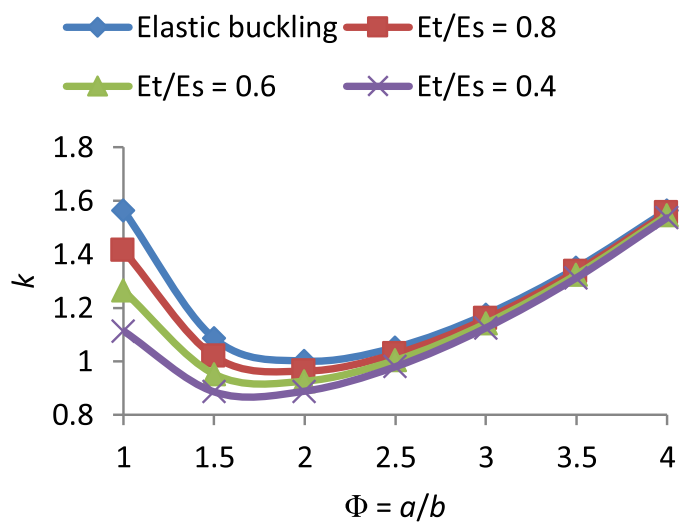

Figure 3. Aspect ratio versus buckling coefficient of SSFS rectangular plate

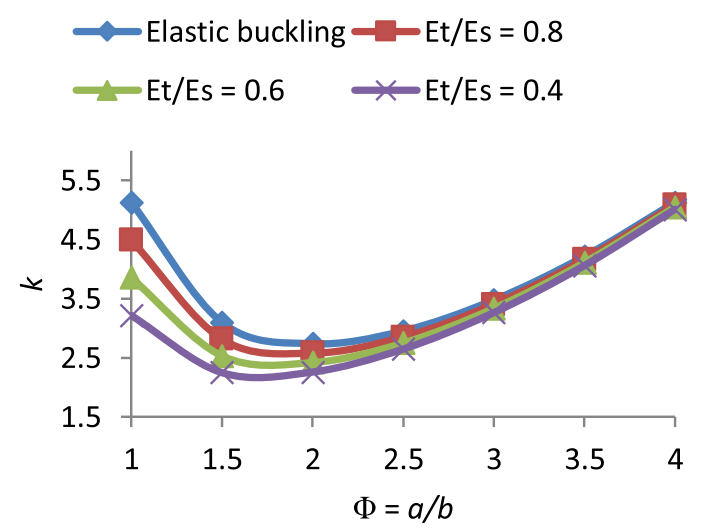

Figure 4. Aspect ratio versus buckling coefficient of CCFC rectangular plate

$$
w=A H
$$

The method for calculating the unknown constants of the buckling curve expression of Eq. (11) for any combination of boundary conditions of rectangular plates are explained in detail by Onwuka et al. [23]. Following similar procedures based on boundary conditions (2) to (7), the characteristic deflection functions of the plate are as follows:

SSFS:

$$
w=A\left[\left(X-2 X^{3}+X^{4}\right)\left(8 Y-4 Y^{3}+Y^{4}\right)\right]
$$

CCFC:

$$
w=A\left[\left(X^{2}-2 X^{3}+X^{4}\right)\left(4 Y^{2}-4 Y^{3}+Y^{4}\right)\right]
$$

SCFS:

$$
w=A\left[\left(1.5 X^{2}-2.5 X^{3}+X^{4}\right)\left(8 Y-4 Y^{3}+Y^{4}\right)\right]
$$

SCFC:

$$
w=A\left[\left(X^{2}-2 X^{3}+X^{4}\right)\left(8 Y-4 Y^{3}+Y^{4}\right)\right]
$$

CSFS:

$$
w=A\left[\left(X-2 X^{3}+X^{4}\right)\left(4 Y^{2}-4 Y^{3}+Y^{4}\right)\right]
$$

CSFC:

$$
w=A\left[\left(1.5 X^{2}-2.5 X^{3}+X^{4}\right)\left(4 Y^{2}-4 Y^{3}+Y^{4}\right)\right]
$$

\section{CRITICAL BUCKLING LOAD}

The governing plastic buckling differential equation of equilibrium of a thin rectangular isotropic plate under inplane uniaxial compressive loading along the $x$-axis is given as follows [24]:

$$
\left(\frac{1}{4}+\frac{3}{4} \frac{E_{t}}{E_{s}}\right) \frac{\partial^{4} w}{\partial x^{4}}+2 \frac{\partial^{4} w}{\partial x^{2} \partial y^{2}}+\frac{\partial^{4} w}{\partial y^{4}}-\frac{N_{x}}{\bar{D}} \frac{\partial^{2} w}{\partial x^{2}}=0
$$

where $E_{t}$ and $E_{s}$ are the tangent and secant modulus, respectively, $N_{x}$ is the in-plane compressive load, and $\bar{D}$ is the plate flexural rigidity in the plastic range. The tangent modulus and secant modulus are both determined by the 
uniaxial stress-strain curve of the material of the plate. In Eq. (19), the flexural rigidity within the plastic portion of the stress-strain curve is expressed as follows [24]:

$$
\bar{D}=\frac{E_{s} t^{3}}{9}
$$

where $t$ is the plate thickness.

A work principle method was applied in [14] using a total work error function. For a single degree of freedom, the buckling load Eq. (21) is obtained:

$$
H=\left(X-2 X^{3}+X^{4}\right)\left(8 Y-4 Y^{3}+Y^{4}\right)
$$

The numerical values of the integrals of the SSFS plate are as follows:

$$
\begin{gathered}
\int_{0}^{1} \int_{0}^{1} H \frac{\partial^{4} H}{\partial X^{4}} \partial X \partial Y=52.7847619 \\
\int_{0}^{1} \int_{0}^{1} 2 H \frac{\partial^{4} H}{\partial R^{2} \partial Q^{2}} \partial X \partial Y=30.19755067
\end{gathered}
$$

where $\Phi$ is the aspect ratio. The aspect ratio is defined in in Eq. (22):

$$
\phi=a / b
$$

Critical buckling occurs when the equilibrium of the plate changes from stable to unstable, and the critical buckling load can be expressed in the form:

$$
N_{x, C R}=\frac{\pi^{2} \bar{D}}{b^{2}} k
$$

where $N_{x C R}$ is the critical inelastic buckling load, and $k$ is the buckling coefficient. The critical load is calculated by first finding the integrals of buckling load Eq. (21) through the application of variational principles. For the SSFS plate, the buckling curve expression from Eqs (12) and (13) is given in Eq. (24):

$$
\begin{aligned}
& \int_{0}^{1} \int_{0}^{1} H \frac{\partial^{4} H}{\partial Y^{4}} \partial X \partial Y=3.779047619 \\
& \int_{0}^{1} \int_{0}^{1} H \frac{\partial^{2} H}{\partial X^{2}} \partial X \partial Y=6.118457978
\end{aligned}
$$

Substituting Eqs (24)-(28) into Eq. (21) yields the expression for the critical inelastic buckling load expressed in Eq. (29).

$$
N_{x, C R}=\frac{\bar{D}}{b^{2}}\left[\frac{\frac{60.4647619}{p^{2}}\left(\frac{1}{4}+\frac{3}{4} \frac{E_{t}}{E_{s}}\right)+30.19755067+3.779047619 p^{2}}{6.118457978}\right]
$$
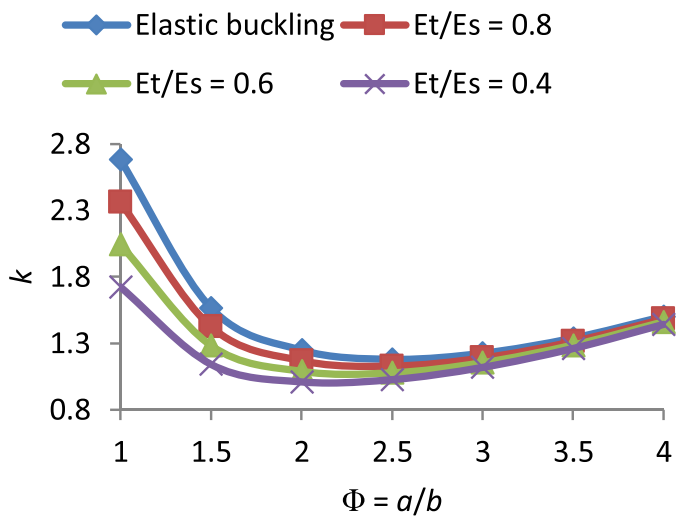

Figure 5. Aspect ratio versus buckling coefficient of SCFS rectangular plate
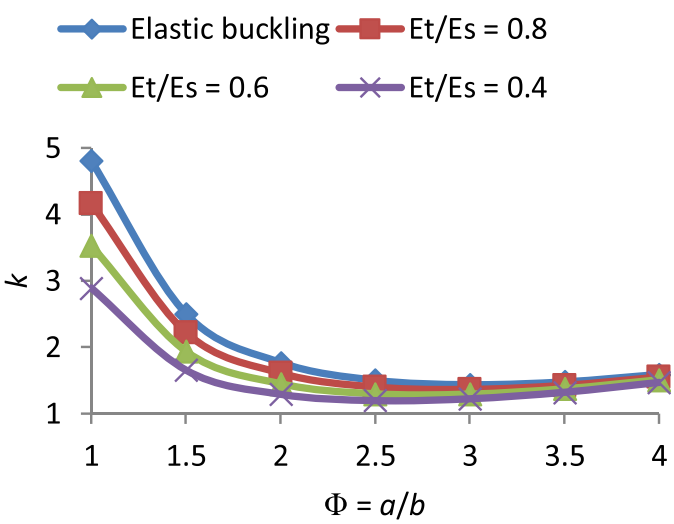

Figure 6. Aspect ratio versus buckling coefficient of SCFC rectangular plate 


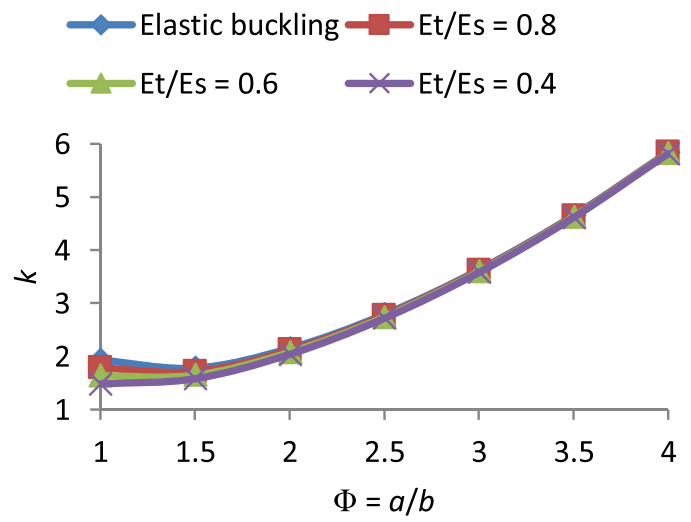

Figure 7. Aspect ratio versus buckling coefficient of CSFS rectangular plate

Expressing Eq. (29) in form of Eq. (23) gives the buckling coefficient of the SSFS plate in Eq. (30).

$\left.k=\frac{1.001292}{\phi^{2}}\left(\frac{1}{4}+\frac{3}{4} \frac{E_{t}}{E_{s}}\right)+0.500069+0.062581 \phi^{2}\right)$

The procedures described from Eqs (24) to (30) are applied to the other rectangular plates. The buckling coefficients of the CCFC, SCFS, SCFC, CSFS and CSFC plates are given in Eqs (31)-(35), respectively.

$$
\begin{aligned}
& k=\frac{4.255490}{\phi^{2}}\left(\frac{1}{4}+\frac{3}{4} \frac{E_{t}}{E_{s}}\right)+0.607927+0.265968 \phi^{2} \\
& k=\frac{2.127745}{\phi^{2}}\left(\frac{1}{4}+\frac{3}{4} \frac{E_{t}}{E_{s}}\right)+0.500069+0.054338 \phi^{2} \\
& k=\frac{4.255490}{\phi^{2}}\left(\frac{1}{4}+\frac{3}{4} \frac{E_{t}}{E_{s}}\right)+0.500069+0.051478 \phi^{2} \\
& k=\frac{1.001292}{\phi^{2}}\left(\frac{1}{4}+\frac{3}{4} \frac{E_{t}}{E_{s}}\right)+0.607927+0.323334 \phi^{2} \\
& k=\frac{2.127745}{\phi^{2}}\left(\frac{1}{4}+\frac{3}{4} \frac{E_{t}}{E_{s}}\right)+0.607927+0.280744 \phi^{2}
\end{aligned}
$$
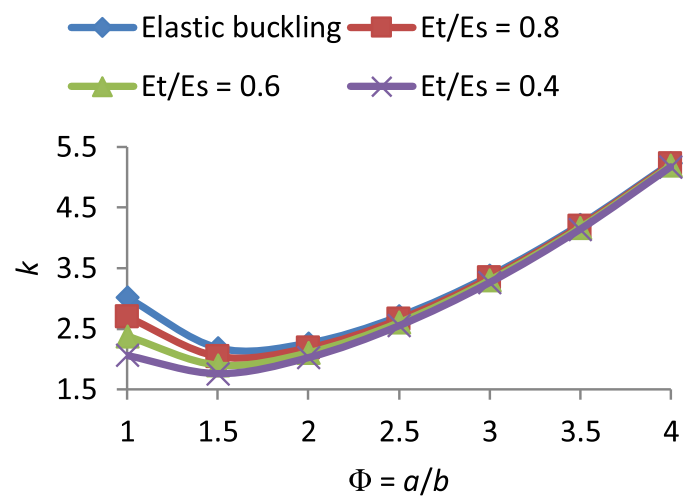

Figure 8. Aspect ratio versus buckling coefficient of CSFC rectangular plate

\section{RESULTS AND DISCUSSION}

The factors that influence the plate buckling coefficient are the aspect ratio and moduli ratio, as can be seen from Eqs. (30)-(35). Thus, buckling coefficients are calculated for various aspect ratios and moduli ratios. The relationship between the buckling coefficient and the aspect ratio are presented from Figs 3 to 8 for the different rectangular plates. A special case exists for a square plate with the aspect ratio being equal to one. Also note that by setting $E_{t}=E_{s}$, the moduli ratio is equal to unity, and the problem is thus reduced to elastic buckling theory. A general trend that can be observed from Figs 3 to 8 is that the reduction in the moduli ratio decreases the buckling coefficient of the plate. Differences in buckling coefficients due to variations in moduli ratio are higher for aspect ratios ranging from 1 to 2 . However, as $k$ approaches 4 , the effect of the moduli ratio on the buckling coefficient reduces significantly.

The aspect ratio significantly influences the buckling coefficient of the plate, especially when the aspect ratio is less than one $(\Phi<1)$. For $\Phi \leq 1$, the values of the buckling coefficient were generally highest for the CCFC plate, followed by SCFC plate. The least values of buckling coefficient for $\Phi \leq 1$ are observed for the SSFS plate. At $\Phi=4$, the CSFS plate had the highest buckling coefficients, while the SCFS produced the least values of buckling coefficient. The least values of buckling coefficient for the SSFS, CCFC and CSFC are observed for $1.5 \leq \Phi \leq 2$. Depending on the actual values of moduli ratio for the SCFS and SCFC, the corresponding lowest values of buckling coefficient lie between 2 $\leq \Phi \leq 3$, whereas those of CSFS exist within $1 \leq \Phi \leq 1.5$. The least buckling coefficient is usually of practical interest because lower values of buckling coefficient will give corresponding lower stresses and loads that will cause buckling.

The relationship between the buckling coefficient, plate thickness, and critical inelastic buckling stress can be derived by substituting the flexural rigidity expression given in Eq. (20) into Eq. (23) and making $\sigma_{x, \mathrm{CR}}$ the subject of the equation. Therefore:

$$
\sigma_{x, C R}=\frac{\pi^{2} k E_{s}}{9}\left(\frac{t}{b}\right)^{2}
$$

where $\sigma_{x, \mathrm{CR}}$ is the critical inelastic buckling stress. In the classical thin plate theory $t / b$ is less than 0.05 (Eq. 36). From Eq. (36), the critical inelastic buckling stresses of a thin rectangular plate having one unloaded free edge can be determined for different arrangements of boundary conditions. It may be observed that the critical buckling mode occurs in mode $m=1$. The critical buckling stress defined by Eq. (36) expresses the limit state criteria for the plastic buckling of the thin rectangular plate. However, the actual values of the secant modulus need to be first known from the uniaxial stress-strain curve of the plate material.

There is shortage of literature for results of inelastic/ plastic buckling of rectangular thin plates with an unloaded free edge. To validate the accuracy of the present study, the solutions are compared with available data found in the 
Table 1. Buckling coefficient of SSFS rectangular thin plate $\left(E_{t} / E_{s}=1\right)$

\begin{tabular}{lcccc}
\hline & \multicolumn{4}{c}{$K$} \\
\cline { 2 - 5 }$\Phi$ & Wang et al. [2] & Iyengar [12] & Nwadike [25] & Present study \\
\hline 0.1 & - & - & 100.3797 & 100.63 \\
0.2 & - & 25.35 & 25.2848 & 25.5349 \\
0.4 & 6.6367 & 6.34 & 6.5181 & 6.7682 \\
0.6 & 3.1921 & - & 3.0539 & 3.3040 \\
0.8 & 1.9894 & 2.16 & 1.8546 & 2.1046 \\
1.0 & 1.4342 & 1.75 & 1.3139 & 1.5639 \\
$\sqrt{ } 2$ & - & 1.43 & - & 1.1259 \\
1.5 & 0.8880 & - & 0.8359 & 1.0859 \\
2 & 0.6979 & 1.47 & 0.7507 & 1.0007 \\
2.5 & 0.6104 & - & - & 1.0514 \\
\hline
\end{tabular}

literature. Table 1 shows the elastic buckling coefficient for uniaxial compression of SSFS rectangular thin plate obtained from different authors. The results of the present study reasonably agree with previous analytical solutions, especially for $\Phi \leq 1$. The results presented in Table 1 are for different material properties; the values of $\mu$ used in Refs. [25] and [12] are 0.25 and 0.3 , respectively. The present study based on [21] applied $\mu=0.5$ in deriving the governing equation, and the results given in Table 1 were set at $E_{t} / E_{s}=1$.

\section{CONCLUSION}

In this study, the inelastic buckling behaviour of uniaxially loaded isotropic thin rectangular plates with an unloaded free edge is investigated using a modified Stowell's technique. The combinations of the boundary conditions are SSFS, CCFC, SCFS, SCFC, CSFS, and CSFC. A unique deflection function was first obtained for each plate using polynomial functions in the form of Taylor-Maclaurin series. The buckling coefficients of each rectangular plate for various values of aspect ratio and moduli ratio were presented. The solutions reasonably agreed with existing results documented in literature. The aspect ratio has a significant influence on the buckling coefficient of the plate, especially when the value of the aspect ratio is less than one $(\Phi<1)$. The effect of moduli ratio on the buckling coefficient decreases as the aspect ratio increases. Inelastic buckling coefficients of thin rectangular plates having a free unloaded edge are presented which can be used to compare other derived solutions of future investigations. The Taylor's series used in this study will be useful in formulating deflection functions of different combinations of boundary conditions whose solutions are difficult to obtain. The technique proposed in this paper may be extended to other loading and boundary conditions, such as plates under biaxial compression and plates with loaded free edges.

\section{REFERENCES}

[1] Aung J. M. (2006), Plastic Buckling of Mindlin Plates. PhD thesis, National University of Singapore.
[2] Wang C. M., Wang C. Y., Reddy J. N. (2005), Exact Solution for Buckling of Structural Members. CRC Press, Boca Raton.

[3] Belinha J., Dinis L. M. J. S. (2006), Elasto-plastic analysis of plates by the element free Galerkin method. Eng. Comput., 23, $525-551$.

[4] Wang X., Huang J. (2009), Elastoplastic buckling analyses of rectangular plates under biaxial loadings by the differential quadrature method. Thin-Walled Struct., 47, 14-20.

[5] Becque J. (2010), Inelastic plate buckling. J. Eng. Mech., 136, 1123-1130.

[6] Komur M. A. (2011), Elasto-plastic buckling analysis for perforated steel plates subjected to uniform compression. Mech. Res. Commun., 38, 117-122.

[7] Maarefdoust M., Kadkhodayan M. (2013), Elastoplastic buckling analysis of plates involving free edges by deformation theory of plasticity. Int. J. Eng. - Trans A: Basics., 26, 421-432.

[8] Fallah N., Parayandeh-Shahrestany A. (2014), A novel finite volume based formulation for the elasto-plastic analysis of plates. Thin-Walled Struct., 77, 153-164.

[9] Kadkhodayan M., Maarefdoust M. (2014), Elastic/plastic buckling of isotropic thin plates subjected to uniform and linearly varying in-plane loading using incremental and deformation theories. Aero. Sci. Technol., 32, 66-83.

[10] Maarefdoust M., Kadkhodayan M. (2014), Elastic/plastic buckling analysis of skew thin plates based on incremental and deformation theories of plasticity using generalized differential quadrature method. Int. J. Eng. - Trans B: Appl., 27, 1277-1286.

[11] Maarefdoust M., Kadkhodayan M. (2015), Elastic/plastic analysis of skew plates under in-plane shear loading with incremental and deformation theories of plasticity by GDQ method. J. Braz. Soc. Mech. Sci. Eng., 37, 761-776.

[12] Iyengar N. G. R. (1988), Structural Stability of Columns and Plates. Ellis Horwood, Chichester.

[13] Ibearugbulem O. M., Oguaghamba O. A., Njoku K. O., Nwaokorie M. (2014), Using line continuum to explain work principle method for structural continuum analysis. Int. J. Innovat. Res. and Dev., 3, 365-370.

[14] Eziefula U. G., Onwuka D. O., Ibearugbulem O. M. (2017), Work principle in inelastic buckling analysis of axially compressed rectangular plates. World J. Eng., 14, 95-100.

[15] Lopatin A. V., Morozov E.V. (2009), Buckling of the SSFF rectangular orthotropic plate under in-plane pure bending. Compos. Struct., 90, 287-294.

[16] Radosavljevic V., Drazic M. (2010), Exact solution for buckling of FCFC stepped rectangular plates. Appl. Math. Model., 34, 3841-3849.

[17] Lopatin A. V., Morozov E. V. (2011), Buckling of the SSCF rectangular orthotropic plate subjected to linearly varying in-plane loading. Compos. Struct., 93, 1900-1909.

[18] Lopatin A. V., Morozov E. V. (2013), Buckling of a uniformly compressed rectangular SSCF composite sandwich plate. Compos. Struct., 105, 108-115.

[19] Lopatin A. V., Morozov E. V. (2014), Approximate buckling analysis of the CCFF orthotropic plates subjected to in-plane bending. Int. J. Mech. Sci., 85, 38-44.

[20] Zureick A. H. (2018), On the buckling of an isotropic rectangular plate uniformly compressed on two simply supported edges and with two free unloaded edges. Thin-Walled Struct., 124, 180-183. 
[21] Glynn J. (2011), Advanced Modern Engineering Mathematics, 4th edition, Palgrave Macmillan, New York.

[22] Ibearugbulem O. M. (2012), Application of a Direct Variational Principle in Elastic Stability Analysis of Thin Rectangular Flat Plates. PhD thesis, Federal University of Technology Owerri.

[23] Onwuka D. O., Ibearugbulem O. M., Eziefula U. G. (2013), Plastic buckling of SSSS thin rectangular plates subjected to uniaxial compression using Taylor-Maclaurin shape function. Int. J. Struct. Civ. Eng. Res., 2, 168-175.

[24] Stowell E. Z. (1948), A unified theory of the plastic buckling of columns and plates. NACA Tech. Rep., 898, 127-137.

[25] Nwadike A. N. (2014), Buckling Analysis of Isotropic Rectangular Plates Using Ritz Method. MEng thesis, Federal University of Technology Owerri. 